\title{
The preparation and characterization of gold-conjugated polyphenol nanoparticles as a novel delivery system
}

This article was published in the following Dove Press journal:

International Journal of Nanomedicine

27 March 2012

Number of times this article has been viewed

\author{
Dar-Shih Hsieh ${ }^{1,2}$ \\ Hsiu-Chin $\mathrm{Lu}^{5}$ \\ Cheng-Cheung Chen ${ }^{1,3}$ \\ Chang-Jer $\mathrm{Wu}^{1, *}$ \\ Ming-Kung Yeh ${ }^{4, *}$ \\ 'Department of Food Science, \\ National Taiwan Ocean University, \\ Keelung, Taiwan; ${ }^{2}$ Division of \\ Urology, ${ }^{3}$ Department of Pathology, \\ Tri-Service General Hospital, \\ ${ }^{4}$ Institute of Preventive Medicine, \\ National Defence Medical Center, \\ Taipei, Taiwan; ${ }^{5}$ Department of \\ Laboratory Medicine, Kaohsiung \\ Medical University, Kaohsiung, Taiwan \\ *These authors contributed equally \\ to this work
}

\begin{abstract}
Nanogold particles are commonly used in nanomedicine. We generated physical nanogold ( $\mathrm{pNG}$ ) conjugated with different ratios of epigallocatechin-3-gallate (EGCG) and evaluated its physicochemical properties, antioxidant activity, and cytotoxicity in vitro as well as anticancer activity in vivo. Results showed that the EGCG-pNG conjugates were successfully prepared at ratios between 23:1 and 23:5, with the percentage of EGCG content increasing with the EGCG:pNG ratio from 23:1 (2.0\% $\pm 0.02 \%)$ to $23: 5(28 \% \pm 0.3 \%)$. EGCG-pNG particles at ratios of 23:1 and 23:5 demonstrated significantly decreased size from 500 to $20 \mathrm{~nm}$ and decreasing zeta potentials of $21 \mathrm{mV}$ to $-22 \mathrm{mV}$, respectively. At a ratio of 23:2.5, the EGCGpNG particles (27\% EGCG, $50 \mathrm{~nm}$ in size, zeta potential of $-8 \mathrm{mV}$ ) showed longer EGCG activity half-life (110 days vs 5 hours), controlled release ( 2 hours vs 30 minutes), and higher antioxidant activity (four times), as well as inhibition of tumor cell growth, than controls. The present study indicated that EGCG-pNG possesses promising therapeutic potential, based on its strong free-radical scavenging and anticancer activities.
\end{abstract}

Keywords: EGCG, nanoparticles, antioxidant, antitumor activity, nanogold

\section{Background}

Nanomedicine involves the utilization of nanotechnology for the benefit of human health and well-being. The use of nanotechnology in various sectors of therapeutics has revolutionized the field of medicine. Metal nanoparticles are new-generation materials that are being widely investigated for biomedical and therapeutic applications, including use in diagnostics, therapeutics, and as biomedical tools for research. In the case of gold nanoparticles (AuNPs), their size, shape, surface chemistry, and optical properties are all properties that can be controlled. They also have unique and very exciting capabilities, such as comparable size with biomolecules and the ability to bind to various molecules, which make them potential candidates for chemical as well as biological applications, such as AuNP-based drug delivery, cell-specific targeting, and controlled drug release. ${ }^{1}$ By taking advantage of their optical-electronic properties, numerous proven applications for AuNPs have shown promise for use in medicinal applications, including plasmon-based labeling and imaging, optical and electrochemical sensing, diagnostics, and therapy for various diseases (eg, cancer). ${ }^{2}$

Many naturally occurring phenolic compounds/substances as well as biotechnological products are receiving continuous attention from the viewpoint of antioxidation and have been shown to be effective antioxidants in biological systems. ${ }^{3}$ Much of the cancer-chemopreventive properties of green tea are mediated by epigallocatechin-3gallate (EGCG), a phenolic compound that induces apoptosis and arrests cell growth. ${ }^{4}$
Institute of Preventive Medicine, National Defense Medical Center, 161 Section 6 Minquan East Road, Taipei I I490, Taiwan Tel +886 2879233 II ext 12860

Fax +88628792 7I54

Email mkyeh2004@gmail.com submit your manuscript | www.dovepress.com

Dovepress

http://dx.doi.org// 0.2147/IJN.\$30060 
Green tea enhances humoral and cell-mediated immunity, decreases the risk of certain cancers, and has certain advantages in treating inflammatory disorders. ${ }^{5}$ EGCG and its derivatives are some of the most important candidates for the development of effective and nontoxic medicines with strong free-radical scavenging and antioxidant actions. ${ }^{6}$ Some studies have demonstrated that EGCG combined with metal ions such as $\mathrm{Fe}^{3+}$ or $\mathrm{Mn}^{2+}$ to form complexes by gallate ring may enhance biological activity. ${ }^{7,8}$

However, no studies have been conducted on EGCG-conjugated nanogold, with the exception of our previous study, ${ }^{9}$ which reported that EGCG particles were physically attached onto the surface of physical nanogold ( $\mathrm{pNG}$ ) particles, confirmed by scanning electron microscopy. The anticancer activity of the EGCG-adsorbed pNG was investigated and confirmed to inhibit tumor cell growth by means of cell apoptosis. The mechanism by which EGCG-pNG mediates tumor apoptosis was discovered to be activation of the caspase cascade through the Bcl-family proteins in the mitochondrial pathway. Additionally, the mechanism by which tumors are suppressed by injecting EGCG-pNG directly into the tumor site is determined to be through downregulation of vascular endothelial growth factor, whereas that by oral administration of EGCG is through reversing immune suppression upon cancer progression. In this study, pNG conjugated with EGCG resulted in the development of therapeutically useful and stable EGCG-pNG. The properties and stability of these particles were evaluated, and their antioxidant and antitumor activity in vivo were determined.

\section{Materials and methods}

\section{Chemicals and apparatus}

The EGCG was purchased from Sinopharm Chemical Reagent (Taipei, Taiwan), $1.6 \mathrm{~nm}$ pNG was purchased from Gold NanoTech (Taipei, Taiwan) and a Panasonic NN-S563JF frequency-conversion microwave oven was purchased from Panasonic (Tokyo, Japan). All other chemicals used were of analytical grade.

\section{High-performance liquid chromatography conditions}

Qualitative EGCG was analyzed by high-performance liquid chromatography (HPLC). A Waters (Milford, MA) HPLC system equipped with an automated gradient controller, 510 pumps, U6K injector, 481 detector, 746 data module, and Waters $\mu$ Bondapak C18 column $(3.9 \times 300 \mathrm{~mm})$ was used for the analysis. Elution was carried out at ambient temperature $\left(25^{\circ} \mathrm{C}\right)$ using $30 \mathrm{mM} \mathrm{NaH}_{2} \mathrm{PO}_{4}$ :acetonitrile (80:20) as a mobile phase at a flow rate of $1.0 \mathrm{~mL} / \mathrm{min}$. UV detection was carried out at $274 \mathrm{~nm}$. Samples were prepared by centrifugation at 14,000 rpm (Sigma 1-14; Sartorius, Goettingen, Germany) for 2 hours. Then, $50 \mu \mathrm{L}$ of supernatant added to $50 \mu \mathrm{M}$ pentoxifylline (as an internal standard) was used for HPLC analysis. All extracts were prepared in triplicate, and each extract was analyzed three times. The EGCG and pentoxifylline retention times were 10.7 and 16.9 minutes, respectively. The EGCG concentration was found to be linear over a range of $0.06-4 \mu \mathrm{g} / \mathrm{mL}$. The correlation coefficient was 0.9996 . The lowest value of the standard curve $(0.056 \mu \mathrm{g} / \mathrm{mL})$ was taken as the limit of quantization. The recovery of EGCG from three concentrations $(0.1,1$, and $4 \mu \mathrm{g} / \mathrm{mL})$ was $80.13 \% \pm 5.14 \%, 87.37 \% \pm 3.51 \%$, and $89.25 \% \pm 2.19 \%$, respectively. The relative standard deviations of accuracy and precision for three different EGCG concentrations $(0.1$, 1 , and $4 \mu \mathrm{g} / \mathrm{mL}$ ) were $5.38 \%, 2.99 \%$, and $1.86 \%$ for intraday analysis and $4.58 \%, 3.03 \%$, and $1.40 \%$ for interday analysis, respectively.

\section{EGCG-pNG particle formulation, purification, and the ratio of EGCG contained in the particles}

A total of $1 \mathrm{~mL}$ of 23-ppm EGCG in $200 \mathrm{mM}$ glycine hydrochloride $(\mathrm{HCl} ; \mathrm{pH} 2.0)$ was mixed with $1 \mathrm{~mL}$ of final concentration $1.0,1.25,2.5$, and $5.0 \mathrm{ppm} 1.6-\mathrm{nm}$ diameter $\mathrm{pNGs}$ in $1 \mathrm{M}$ Tris- $\mathrm{HCl}$ (pH 8.5) and ultrasonicated for 5 minutes at room temperature for the preparation of EGCG-pNG. The EGCG-pNG samples were then centrifuged at 14,000 rpm for 2 hours. The nanoparticles were cleaned by centrifugation and resuspended in distilled water three times to remove the EGCG. The free EGCG was determined by the above HPLC method. The concentration of pNG was calculated based on ultravioletvisible spectrophotometer (DU-640; Beckman Coulter, Brea, CA) analysis, and ultraviolet-visible detection was carried out at $520 \mathrm{~nm}$. The 5-ppm concentration of $1.6 \mathrm{~nm}$ pNG was used to make a standard curve. The concentration of pNG was calculated based on the data that $1.6 \mathrm{~nm} \mathrm{pNG}$ has an absorption of 0.8 at $520 \mathrm{~nm}$ when the concentration is $5 \times 10^{13} \mathrm{~mL}^{-1}$. We calculated the $\mathrm{pNG}$ concentration by following the formula: $C_{\mathrm{pNG}}=5 \times 10^{13} \mathrm{~mL}^{-1} \times A / 0.8(A$ is the absorption of the sample at $520 \mathrm{~nm}$ ). Each sample was assayed in triplicate. The fraction of EGCG contained in the EGCG-pNG was calculated by dividing the total EGCG amount by the sum of the total pNG and EGCG amounts. 


\section{EGCG-pNG particle properties}

Nanoparticle size was characterized by photon correlation spectroscopy (Zetasizer Nano ZS; Malvern Instruments, Malvern, UK). All measurements were done at a wavelength of $633 \mathrm{~nm}$ at room temperature with an angle of detection of $173^{\circ}$. Raw data were subsequently correlated to mean hydrodynamic size by cumulant analysis (Z-average mean). The zeta potential of all nanoparticles was analyzed by laser Doppler anemometry (Zetasizer Nano ZS). All samples were run in triplicate, and the mean averages and standard deviations were calculated. Samples were also examined using a Philips (Amsterdam, Netherlands) CM10 transmission electron microscope operating at an accelerating voltage of $80 \mathrm{kV}$. Images were recorded digitally with a Gatan (Munich, Germany) MultiScan 791 CCD camera using Gatan's Digital Micrograph 3.1 software pack.

\section{Stability of EGCG-pNG at various $\mathrm{pH}$ levels}

A total of $1 \mathrm{~mL}$ of EGCG-pNG (23:2.5) was sealed in an ampoule and placed in a digitally controlled incubator to equilibrate at $40^{\circ} \mathrm{C}$ (Fisher Scientific, St Louis, MO). The $\mathrm{pH}$ of EGCG-pNG dispersion was adjusted using $0.04 \mathrm{M}$ trisodium citrate and $0.04 \mathrm{M}$ citric acid $(\mathrm{pH} 2.32,2.77,3.60$, 4.46, 5.67, and 6.12) using a calibrated $\mathrm{pH}$ meter (Delux $\mathrm{pH}$ Meter 101; Adarsh Scientific Industries, Haryana, India). To determine the dissociation of free EGCG, the samples were analyzed at predetermined days $(0,1,3$, and 7$)$. The determination of the percentage of free EGCG was carried out by a previously determined validated HPLC method. Each sample was assayed in triplicate.

Half-lives $\left(t_{1 / 2}\right)$ were calculated for the percentage of EGCG-pNG remaining for all the data points from 0 to 7 days, which followed zero-order kinetics. The dissociation rate constant $(\mathrm{k})$ was calculated by the slope, and the following equation was used to calculate the half-life: $\mathrm{k}=0.693 \mathrm{t}_{1 / 2}$

\section{In vitro release test}

In vitro release of EGCG from the EGCG-AuNPs (23:2.5) was determined using a dialysis device with a $100 \mathrm{KDa}$ molecular weight cut-off in $150 \mathrm{~mL}$ of $\mathrm{HCl}$ solution ( $\mathrm{pH} 1.2$ ) at $36^{\circ} \mathrm{C}$ following the method published previously. An accurately measured amount of various particle formulations was transferred to the dialysis bag and placed in a receiving compartment containing $120 \mathrm{~mL} \mathrm{HCl}$ solution (pH 1.2). Control bags were prepared and tested in parallel. Each control bag contained $0.1 \mathrm{mg}$ EGCG. The two ends of the dialysis bag were tightly bound with thread. The bags were hung inside a conical flask with the help of a glass rod so that the portion of the dialysis bag with the formulation dipped into the $\mathrm{HCl}$ solution. The flask was kept on a magnetic stirrer, and stirring was maintained at $100 \mathrm{rpm}$ at $36^{\circ} \mathrm{C}$ with a thermostatic control. At predetermined time intervals, the release medium was withdrawn for analysis. The EGCG release by the particles was calculated by subtracting the initial amount of EGCG in the particles from the amount at the time of release.

\section{Determination of the antioxidant activity of EGCG:pNG at a ratio of 23:2.5}

Superoxide radicals ${ }^{6}$ were generated in $3 \mathrm{~mL}$ Tris- $\mathrm{HCl}$ buffer (16 mM, pH 8.0), which contained $78 \mu \mathrm{M}$ NADH (reduced form), $50 \mu \mathrm{M}$ nitroblue tetrazolium, and $10 \mu \mathrm{M}$ phenazin methosulfate as well as $2 \mathrm{~mL}^{-1}$ of EGCG (23 ppm), pNG (2.5 ppm), EGCG-pNG (23:2.5 ppm), or $\alpha$-tocopherol (vitamin E, $400 \mathrm{IU}$ ) as a control. The color reaction generated by superoxide radicals and nitroblue tetrazolium was detected by monitoring the absorbance at $560 \mathrm{~nm}$. In the essential control, NADH was substituted with Tris-HCl buffer.

Hydroxyl radicals were generated in sodium phosphate buffer $(150 \mathrm{mM}, \mathrm{pH} 7.4)$, which contained $0.15 \mathrm{mM} \mathrm{FeSO}_{4}$ EDTA, $2 \mathrm{mM}$ sodium salicylate, and $6 \mathrm{mM} \mathrm{H}_{2} \mathrm{O}_{2}$ as well as $2 \mathrm{~mL}^{-1}$ of EGCG (23 ppm), pNG (2.5 ppm), EGCG-pNG (23:2.5 ppm), or vitamin E (400 IU) as a control. In the essential control, sodium phosphate buffer replaced $\mathrm{H}_{2} \mathrm{O}_{2}$. The solutions were incubated at $37^{\circ} \mathrm{C}$ for 1 hour and detected by monitoring the absorbance at $510 \mathrm{~nm}$.

Effects of EGCG-pNG (23:2.5 ppm) on $\mathrm{H}_{2} \mathrm{O}_{2}$-induced hemolysis of rat erythrocytes and lipid peroxidation in rat liver microsomes were evaluated. Erythrocytes were separated by centrifugation from blood samples of male Wistar rats and resuspended in physiological saline to a final concentration of $0.5 \%$. A total of $2 \mathrm{~mL}$ of reacting solution, which contained $1 \mathrm{~mL}$ erythrocyte suspension and $100 \mathrm{mM}$ $\mathrm{H}_{2} \mathrm{O}_{2}$ as well as $2 \mathrm{~mL}^{-1}$ of EGCG (23 ppm), pNG (2.5 ppm), EGCG-pNGs (23:2.5 ppm), or vitamin E (400 IU) as a control, was incubated at $37^{\circ} \mathrm{C}$ for 1 hour, diluted with four volumes of physiological saline, and centrifuged at $1000 \times \mathrm{g}$ for 10 minutes. The supernatant was assayed by monitoring its absorbance at $414 \mathrm{~nm}$. The essential control contained only the erythrocyte suspension in $2 \mathrm{~mL}$ of solution.

The percentage of antioxidant activity in the samples and the relative time of vitamin $\mathrm{E}$ antioxidant activity were evaluated according to the following formula: inhibition rate $(\%)=\left(A_{0}-A\right) /\left(A_{0}-A_{e}\right) \times 100 \%$, relative to vitamin $\mathrm{E}$ 
antioxidant activity $=\mathrm{S}_{(\mathrm{IR} \%)} / \mathrm{E}_{(\mathrm{IR} \%)}$; where $\mathrm{A}_{0}$ is the absorbance of the free-radical generation system, $\mathrm{A}$ is the absorbance of the test sample, $\mathrm{A}_{\mathrm{e}}$ is the absorbance of the essential control, $\mathrm{S}_{(\mathrm{IR} \%)}$ is the test sample inhibition rate (\%), and $\mathrm{E}_{(\mathrm{IR} \%)}$ is the vitamin $\mathrm{E}$ inhibition rate (\%).

\section{In vitro antitumor study}

For cell-viability analysis, $1.5 \times 10^{4}$ cells resuspended in $100 \mathrm{~mL}^{-1}$ medium were plated into each well of a 96-well plate for 24 hours. The cells were then treated with different concentrations of native EGCG (11.5 and 23 ppm), pNG (1 and $2.5 \mathrm{ppm}$ ), or EGCG-pNG (23:1 and 23:2.5 ppm) for 24 and 48 hours. Cell proliferation was monitored using the CellTiter 96 AQueous One Solution Assay (Promega, Madison, WI) and performed according to the manufacturer's instructions. Briefly, after treatment, $20 \mathrm{~mL}^{-1}$ of the combined 3-(4,5-dimethylthiazol-2-yl)-5-(3-carboxymethoxyphenyl)2-(4-sulfophenyl)-2H-tetrazolium inner salt (MTS) and phenazine methosulfate solution was added into the wells containing $100 \mathrm{~mL}^{-1}$ of culture medium and incubated for 40 minutes at $37^{\circ} \mathrm{C}$ in a humidified $5 \% \mathrm{CO}_{2}$ atmosphere. The absorbance at $490 \mathrm{~nm}$ was recorded using an enzyme-linked immunosorbent assay plate reader.

Cell viability was calculated using the following formula: cell viability $(\%)=($ Abs of treated cells - Abs of free medium with EGCG)/(Abs of control untreated cells - Abs of free medium alone) $\times 100$. The viability of cells left untreated was normalized to $100 \%$. Six experimental sets were carried out, and each set was performed in triplicate.

\section{Tumor model and experiment protocol}

Female $\mathrm{C} 3 \mathrm{H} / \mathrm{He}$ mice aged 6-8 weeks were housed in a climate-controlled room $\left(24^{\circ} \mathrm{C}, 50 \% \pm 10 \%\right.$ relative humidity, 12-hour-light/12-hour-dark cycle, autoclaved bedding) with food (Labdiet 5001; Purina, St. Louis, MO) and water ad libitum. The Institutional Animal Care and Use Committee of the National Defense Medical Center in Taipei approved all experiments, and all animal work conformed to their guidelines for the humane care of animals. MBT-2 tumor cells $\left(5 \times 10^{5}\right)$ in a volume of $50 \mu \mathrm{L}$ were injected subcutaneously into the right dorsal flank of the mice. Following inoculation with tumor cells or phosphate-buffered saline, body mass, food intake, and tumor size were measured four times weekly. Tumor growth was assessed by the measurement of two bisecting diameters in each tumor using calipers, and the tumor volume was calculated using the following equation: tumor volume $\left(\mathrm{mm}^{3}\right)=$ width $\times$ length $^{2} / 2$. Tumor volumes after treatment were divided by volumes before chemotherapy and are expressed as tumor-volume-reduction ratios (TVRs). ${ }^{10}$ During the exponential growth phase, the tumor-volume-doubling time $(\mathrm{Td})$ was calculated using the following formula ${ }^{11}: \mathrm{Td}=\mathrm{t} \times[\log 2 /(\log \mathrm{Vb}-\log \mathrm{Va})]$, where $\mathrm{Va}$ is tumor volume at initial and $\mathrm{Vb}$ that at $\mathrm{t}$ days after Va.

Experiments were designed to test the drug $(2 \mathrm{mg} / \mathrm{mice}$ EGCG or $2 \mathrm{mg} /$ mice EGCG-pNG [23:2.5]) effects. Drugs were administered orally every other day before MBT- 2 cell implantation, whereas in the treatment group administration started at 14 days after tumor implantation with a palpable nodule.

\section{Statistical analysis}

Data were expressed as the mean \pm standard error of the mean. Statistical differences were assessed with the Student's $t$-test. Statistical significance was set at $P<0.05$.

\section{Results and discussion Evaluation of EGCG-loaded pNG}

In this work, pNG particles were characterized via surface plasmon resonance at $\sim 512 \mathrm{~nm}$ in the ultraviolet-visible spectrum, which confirmed the formation of spheroid nanogold in solution. This was further confirmed by transmission electron microscope analysis indicating that $96 \%$ of the nanogold particles had an approximate size of $1.6 \mathrm{~nm}$ with a nearly uniform size distribution (Figure 1). After the successful synthesis of stable pNG, we selected EGCG for loading on synthesized pNG for drug delivery to tumor cells. Percentage loading of EGCG on $\mathrm{pNG}$ was determined based on EGCG content in the EGCGpNG particles, and the percentage of EGCG content was observed to have increased with an increasing EGCG:pNG ratio from $23: 1(2.0 \% \pm 0.02 \%)$ to $23: 5(28 \% \pm 0.3 \%)$ (Figure 2). Figure 2 shows that changes in the EGCG:pNG ratio altered the effective diameter and zeta potential of the conjugate particles; particle size and zeta potential decreased significantly with increasing EGCG:pNG ratios from 23:1 to 23:5. Freshly prepared EGCG-pNG at a ratio of 23:1 were around $500 \mathrm{~nm}$ in size and had a zeta potential of $21 \mathrm{mV}$. The EGCG-pNG particles at a ratio of 23:2.5 contained 27\% EGCG conjugate, were around $50 \mathrm{~nm}$ in size, and had a zeta potential of $-8 \mathrm{mV}$; these particles were used for further study. In our previous report, ${ }^{9}$ EGCG particles were shown to be physically attached to the surface of $\mathrm{pNG}$ particles. In general, particle aggregation is less likely to occur for charged particles with an optimum zeta potential $( \pm 30 \mathrm{mV})$ due to electrostatic repulsion. ${ }^{12}$ 


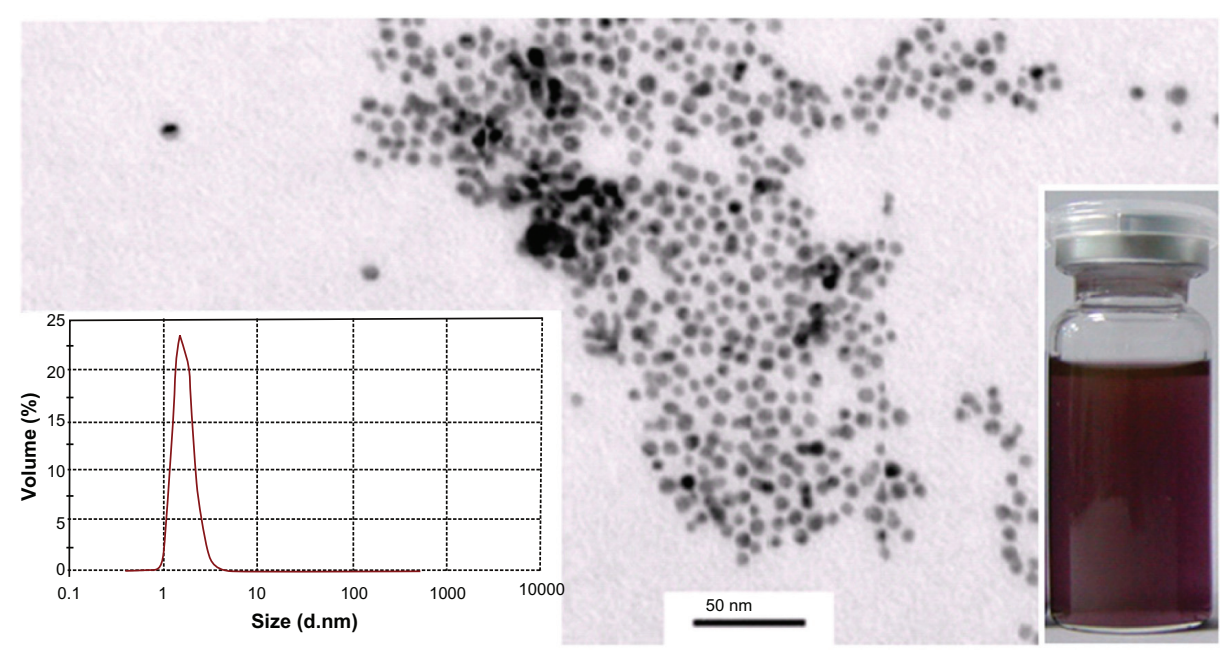

Figure I Transmission electron microscopy analysis of $\mathrm{pNG}$ particles.

Note: Right-inset photograph shows the light-purple color of pNG dispersion, and the left inset shows the dominant peak between I.6 nm in the particle-size distribution graph generated by photon correlation spectroscopy.

Abbreviation: pNG, physical nanogold.

The size of nanoparticles used in a drug-delivery system should be large enough to prevent their rapid leakage into blood capillaries but small enough to escape capture by fixed macrophages that are lodged in the reticuloendothelial system, such as the liver and spleen. The size of the sinusoid in the spleen and fenestra of Kupffer cells in the liver varies from 150 to $200 \mathrm{~nm},{ }^{13}$ and the size of gap junctions between endothelial cells of the leaky tumor vasculature may vary from 50 to $600 \mathrm{~nm} .{ }^{14}$ Sonavane and colleagues also reported 15- to 50-nm AuNPs widespread in organs of mice through intravenous administration. ${ }^{15}$ Consequently, EGCG-pNG nanoparticles up to $50 \mathrm{~nm}$ in size should be able to reach tumor tissues by passing through these two vascular structures.

\section{Stability over wide range of $\mathrm{pH}$ conditions}

For varied therapeutic and biomedical applications, we performed stability analysis of EGCG-pNG (23:2.5) by monitoring EGCG by HPLC over a reasonable period of time and under $\mathrm{pH}$ conditions ranging from 2.32 to 6.12 . Figure 3 shows the dissociation patterns of EGCG from EGCG-pNG (23/2.5) after incubation at varied $\mathrm{pH}$ at $40^{\circ} \mathrm{C}$ for 7 days. The results indicated that EGCG-pNG $(23: 2.5)$

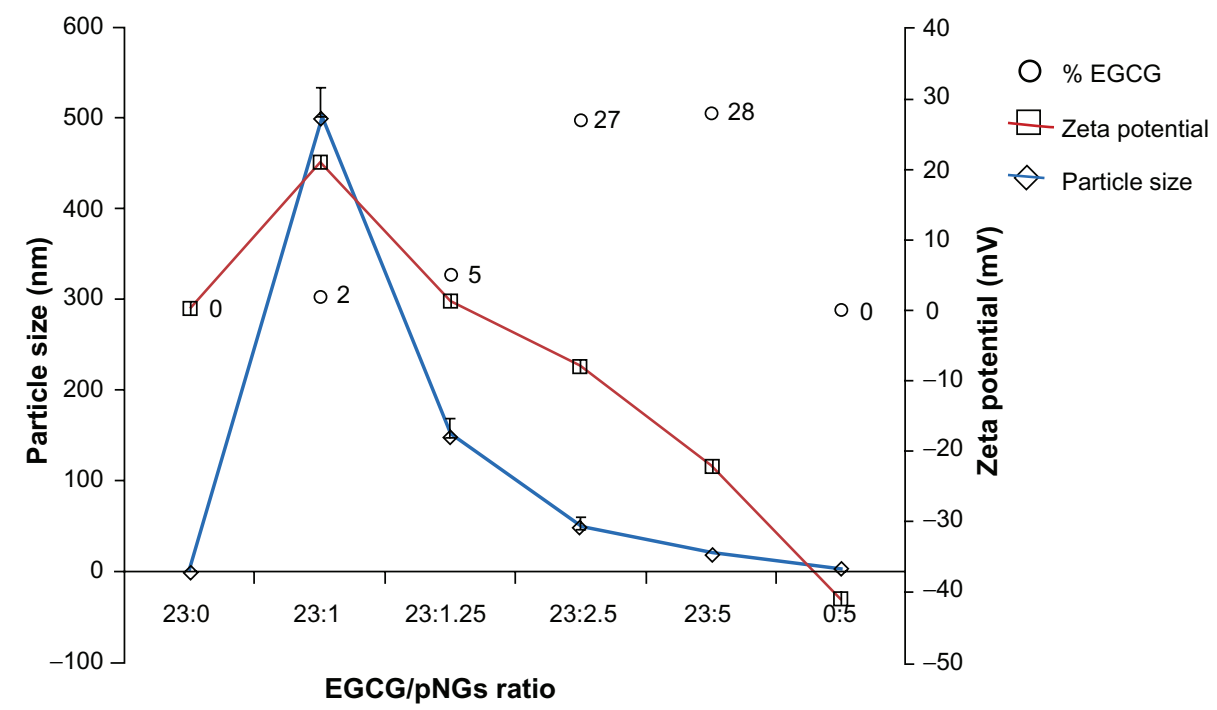

Figure 2 The characteristics of EGCG-pNG particles show the percentage of EGCG capped on pNGs (O), particle size $(\diamond)$, and zeta potential ( $\square)$ at different ratios of EGCG:pNG (23:0, 23:1, 23:1.25, 23:2.5, 23:5, and 0:5).

Abbreviations: EGCG, epigallocatechin-3-gallate; pNG, physical nanogold. 
A
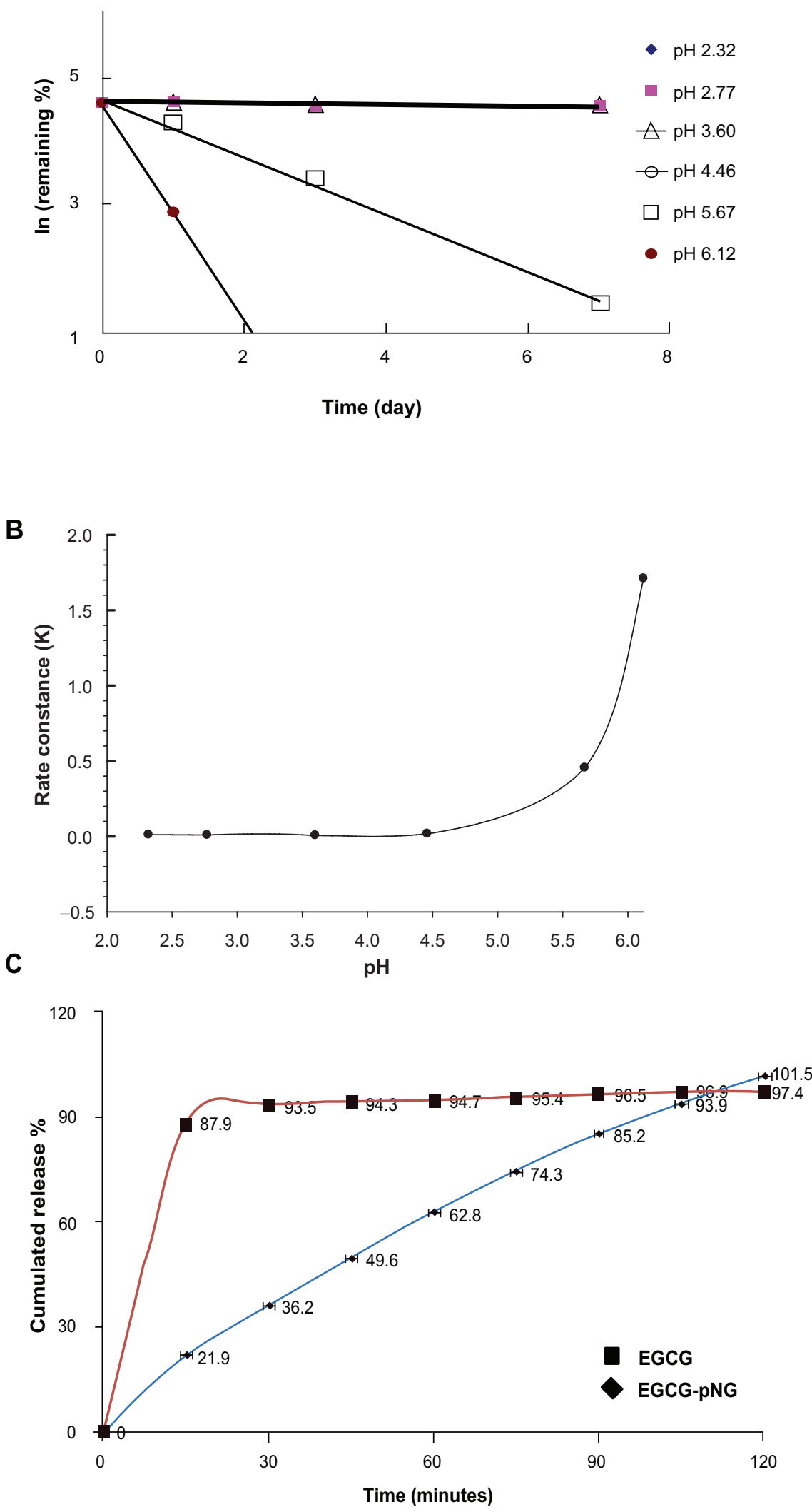

Figure 3 (A) The influence of pH on EGCG-pNG dissociation over time; (B) the relationship with the dissociation rate constant of EGCG-pNG; (C) the release amount of EGCG from the release profile of EGCG-pNG particles in $\mathrm{HCl}$ solution $\left(\mathrm{pH} \mathrm{I.2)}\right.$ ) at $36^{\circ} \mathrm{C}$.

Notes: $\square$, free EGCG; $\diamond$, EGCG-pNG.

Abbreviations: EGCG, epigallocatechin-3-gallate; $\mathrm{HCl}$, hydrochloride; $\mathrm{pNG}$, physical nanogold. 
was easily dissociated into EGCG at pH 6.12 and 5.67. Based on the slope of the plot, the $k$ and $t_{1 / 2}$ of EGCG dissociation from EGCG-pNG $(23: 2.5)$ were calculated. The $\mathrm{k}$ and $\mathrm{t}_{1 / 2}$ for $\mathrm{pH} 2.32,2.77,3.60,4.46,5.67$, and 6.12 were $0.0118,0.0097,0.0062,0.018,0.4553$, and 1.7109 (1/day) and 58.66, 71.4, 110.95, 38.43, 1.52, and 0.41 (day), respectively. Such significant changes at a $\mathrm{pH}>4$ indicated a greater instability of EGCG-pNG compared to borohydrate or citrate-reduced $\mathrm{pNG}$ aggregates. ${ }^{16}$ In our study, nanogold significantly increased the stability of EGCG with a $t_{1 / 2}$ of 110 days compared to EGCG alone at 4-5 hours; similar results were reported by Chen et al. ${ }^{17}$ However, EGCG-pNG (23:2.5) particles were highly stable over a reasonable period of time at a $\mathrm{pH}<4$ (Figure 3B). EGCG-pNG was able to stably endure acidic conditions, which may be suitable to prevent gastric acid decomposing the EGCG, because $\mathrm{pH}$ values of 1.7 have been measured following fasting, and duodenal $\mathrm{pH}$ as high as 6.1 has been reported. ${ }^{18,19}$

\section{The EGCG-release profiles of EGCG-pNG}

EGCG release was investigated by studying release of encapsulated EGCG from EGCG-pNG $(23: 2.5)$ and comparing it with that of free EGCG. Figure 3C illustrates the release profile of EGCG, indicating a prolonged retention time for EGCG-pNG (23:2.5) particles as compared to free EGCG. The initial burst release $(36.2 \%$ at 0.5 hours) of EGCG-pNG (23:2.5) particles was followed by a continued and controlled release phase of $101.5 \%$ at 2 hours.

\section{Antioxidant activities}

Figure 4 shows the superoxide radical scavenging effect, hydroxyl radical effect, and the effects of EGCG-pNG (23:2.5 ppm) on $\mathrm{H}_{2} \mathrm{O}_{2}$-induced hemolysis of rat erythrocytes and lipid peroxidation in rat liver microsomes. The EGCG, pNG, and EGCG-pNG (23:2.5 ppm) conjugates showed significant superoxide radical scavenging activity compared to vitamin E alone (Figure 4). EGCG, a good antioxidant for radical-initiated peroxidation of rat liver microsomes, has been noted by Cai et al. ${ }^{3}$ Also, Dai et al demonstrated that the mixture of the green tea polyphenol, vitamin $\mathrm{E}$, and vitamin $\mathrm{C}$ can act synergistically to protect against lipid peroxidation. ${ }^{6}$ In the process of $\mathrm{H}_{2} \mathrm{O}_{2}$-induced erythrocyte hemolysis, $\mathrm{H}_{2} \mathrm{O}_{2}$ reacts with $\mathrm{Fe}^{2+}$ in erythrocytes to form hydroxyl free radicals, which induces substantial hemolysis. As such, the results of this erythrocyte hemolysis experiment were consistent with that of the hydroxyl free-radical scavenging experiment. Lipid peroxidation is a complex process. It involves the formation and propagation of lipid peroxide and the eventual destruction of membrane lipids, producing breakdown products such as malondialdehyde in microsomes. In our study, we adopted the $\mathrm{Fe}^{2+} /$ vitamin $\mathrm{E}$ system to induce the production of microsomal peroxyl radicals. EGCG-pNG particles demonstrated the strongest ability (about fourfold greater than native cells) to scavenge lipid peroxyl radicals.

\section{In vitro antitumor effect of EGCG-pNG}

In order to demonstrate the antitumor effect of the EGCG/ pNG (23:2.5 ppm) conjugates in vitro, we performed

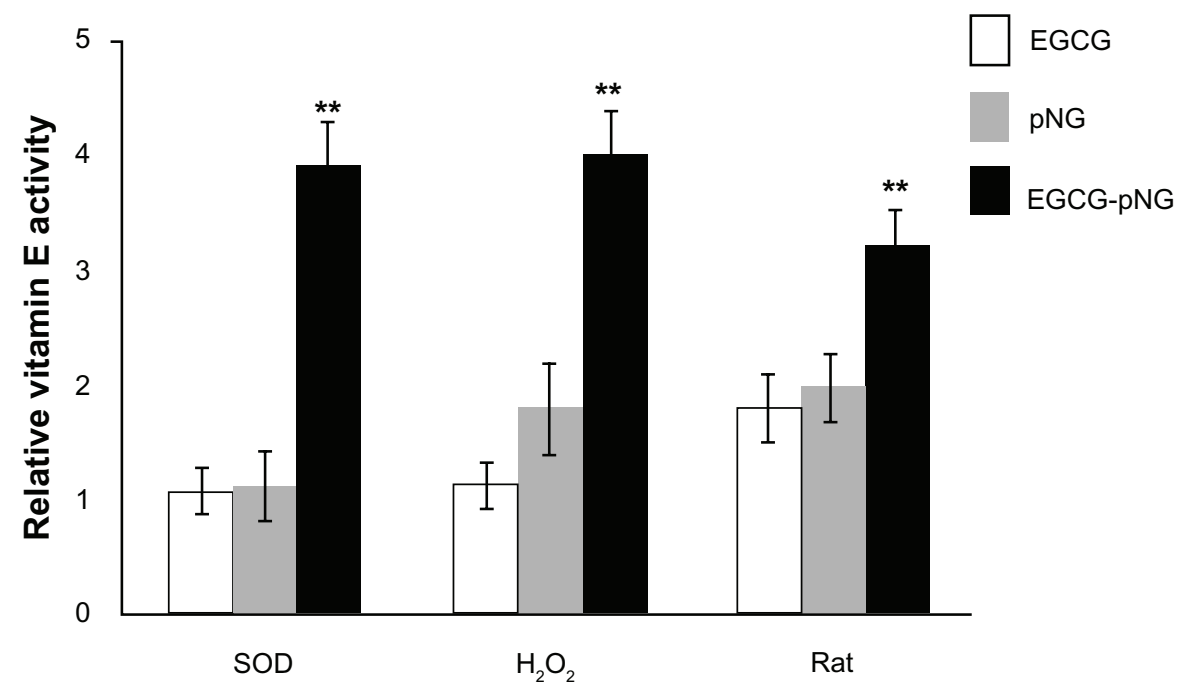

Figure 4 Antioxidant effects of EGCG $(\square)$, pNG ( $\square$ ), and EGCG-pNG 23:2.5 ( $\square$ ) on superoxide radicals, hydroxyl radicals $\left(\mathrm{H}_{2} \mathrm{O}_{2}\right)$, and $\mathrm{H}_{2} \mathrm{O}_{2}$-induced hemolysis of rat erythrocytes and lipid peroxidation in rat liver microsomes.

Note: **P $<0.01$.

Abbreviations: EGCG, epigallocatechin-3-gallate; pNG, physical nanogold. 
cytotoxicity studies of native EGCG (11.5 and 23 ppm), pNG (1 and $2.5 \mathrm{ppm}$ ), and EGCG/pNG (23:1 and 23:2.5 ppm) on MBT-2 cells using an in vitro MTS assay method. At the end of 24 and 48 hours, we observed that native EGCG, pNG, and EGCG/pNG conjugates decreased cell viability (Figure 5). We first examined whether pNG and EGCG had any effect on MBT-2 proliferation. Compared at 24 and 48 hours, a slight concentration-dependent increase in the cytotoxicity of pNG alone was observed, as well as a significant concentration-dependent increase in the cytotoxicity of EGCG. Importantly, where proliferation inhibition was increased with increasing concentrations of pNG, after 48 hours pNG was less toxic than at 24 hours. At the end of 48 hours, the decrease in cell viability with native EGCG and pNG alone in the concentration range studied was found to be $25 \%-12.5 \%$ and $85 \%-80 \%$, respectively. It was interesting to note a significantly increased cytotoxicity for the varied ratios of EGCG-pNG particles as compared to native EGCG or pNG alone $(P<0.0001)$ (Figure 5). Venkatpurwar et al prepared AuNPs capped with porphyran, and these particles exhibited enhanced cytotoxicity in human glioma cells (LN-229), as compared with native porphyran. ${ }^{20}$ They also reported on AuNPs utilized as carriers for the delivery of the anticancer drug doxorubicin hydrochloride, and the doxorubicin-loaded AuNPs demonstrated higher cytotoxicity towards LN-229 cells, as compared with an equal dose of native doxorubicin solution. ${ }^{20}$ Functionalized AuNPs capped with 3-mercaptopropionic acid have also been fabricated, and the effects of these AuNPs on two leukemia cell lines, adriamycin-sensitive K562 cells and adriamycin-resistant K562/A02 cells, have been assayed. ${ }^{21}$ Guo et al demonstrated that these functionalized AuNPs could increase the hydrophobicity of both cell suspensions, greatly decrease the peak potential of both cell lines, and facilitate the cellular uptake of the anticancer drug $\mathrm{As}_{2} \mathrm{O}_{3}$ into K562 leukemia cells. ${ }^{21}$ Thus, AuNPs can inhibit the function of P-glycoprotein to improve the relevant drug accumulation in drug-resistant cancer cells and enhance the cytotoxicity suppression of $\mathrm{As}_{2} \mathrm{O}_{3}$. Chen et al reported the intracellular accumulation of EGCG-conjugated AuNPs was due to AuNP-mediated endocytosis. ${ }^{22}$ In our previous report, ${ }^{9}$ we investigated the mechanism of EGCG- and pNG-mediated cell apoptosis. We showed that MBT-2 cells treated with EGCG or $\mathrm{pNG}$ were arrested in the $\mathrm{S}$ phase of the cell cycle. $\mathrm{Bcl}-\mathrm{XL}$, Bad, and Bax are likely connected with EGCG- and pNG-mediated MBT-2 cell apoptosis. The pNG showed more arrest in the $\mathrm{S}$ phase than the growth inhibition in the antiapoptoic protein BcL-XL. Improvement in cytotoxicity of EGCG/pNG may be attributed to the greater uptake potential of pNG by endocytosis or enhanced retention permeability, as compared to native EGCG. ${ }^{22,23}$ The increase in cytotoxicity of EGCG/pNG may be due to the enrichment in the internalized

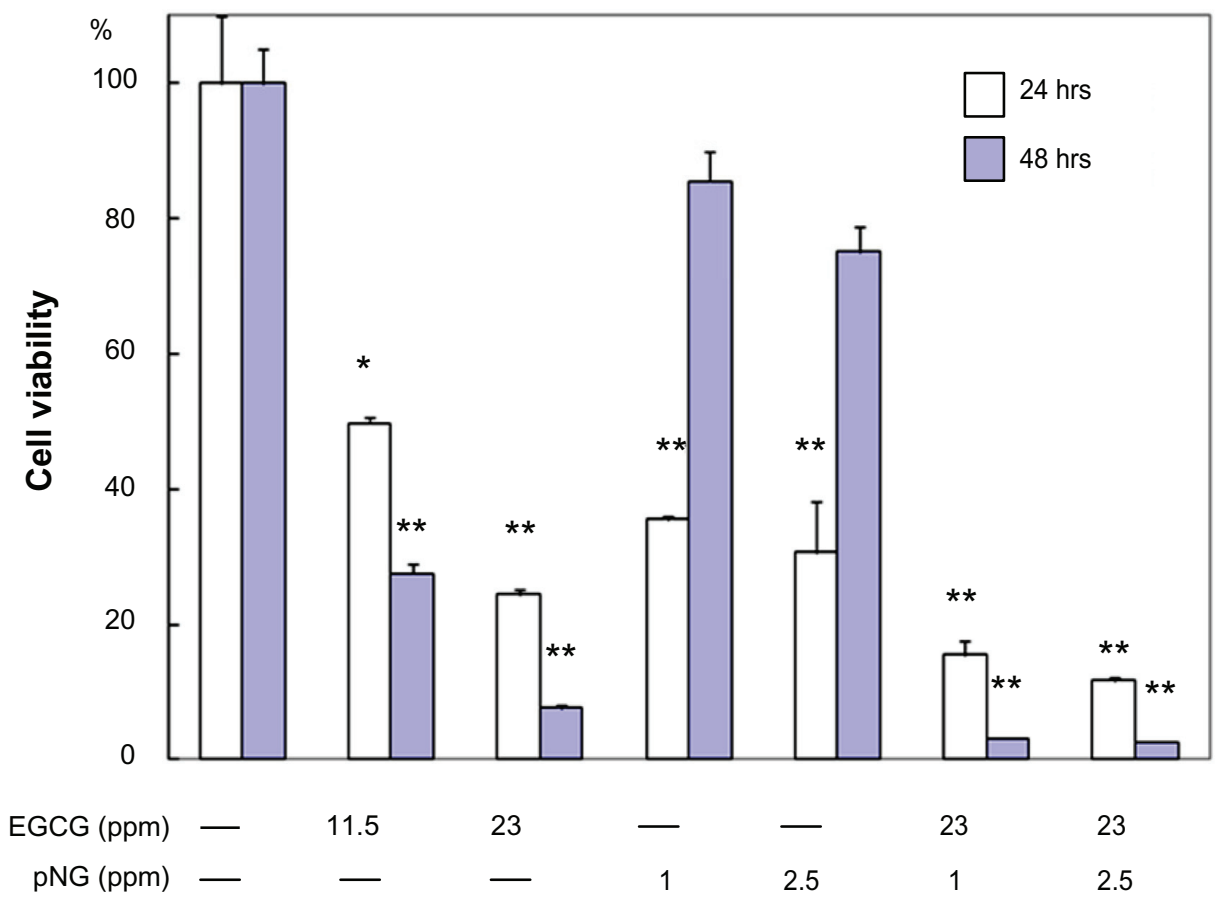

Figure 5 Cell viability of MBT-2 cells treated with EGCG and/or PNG at different concentrations over 24 and 48 hours. Notes: $* p<0.5$, $* * p<0.01$.

Abbreviations: EGCG, epigallocatechin-3-gallate; pNG, physical nanogold. 
EGCG conjugate by an endocytosis mechanism, as compared to the passive diffusion mechanism of native EGCG.

\section{In vivo antitumor activity of EGCG-pNG}

We tested the efficacy of EGCG-pNG to inhibit MBT-2 tumor growth in vivo in a $\mathrm{C} 3 \mathrm{H} / \mathrm{He}$ mouse model. The EGCG and EGCG-pNG conjugates (pretreated or treated groups) were orally or intraperitoneally administered to assess their antitumor activities in vivo (Figure 6). Our results revealed that tumor-volume ratio was reduced in mice receiving either
EGCG alone or receiving EGCG-pNG in both the pretreated or treated groups (Figure 6A). EGCG and EGCG-pNG both demonstrated significant antitumor effects in terms of tumor-double time compared to the control group, which showed 2.7-9.84 days (Figure 6B). More importantly, tumor volume in the EGCG-pNG group was significantly less compared with that in the control group $(P<0.05)$. A reduction in angiogenesis and tumor volume was also observed (Figure 6C). Mice harboring MBT-2 tumors treated with EGCG-pNG developed less edema than mice treated

A

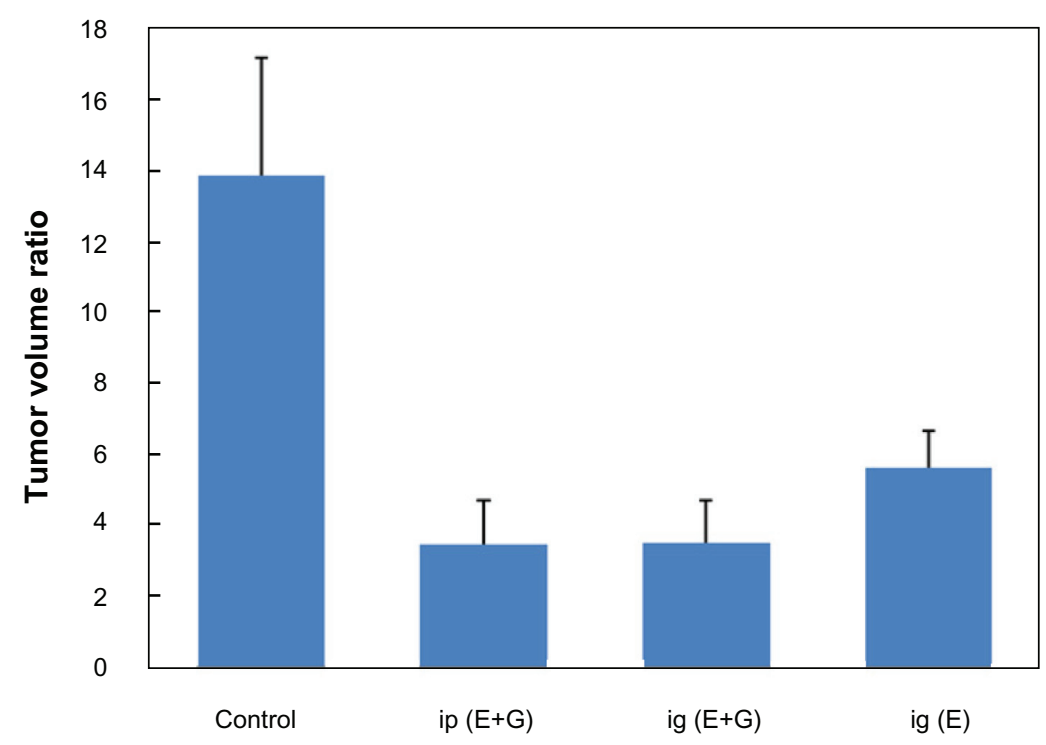

B

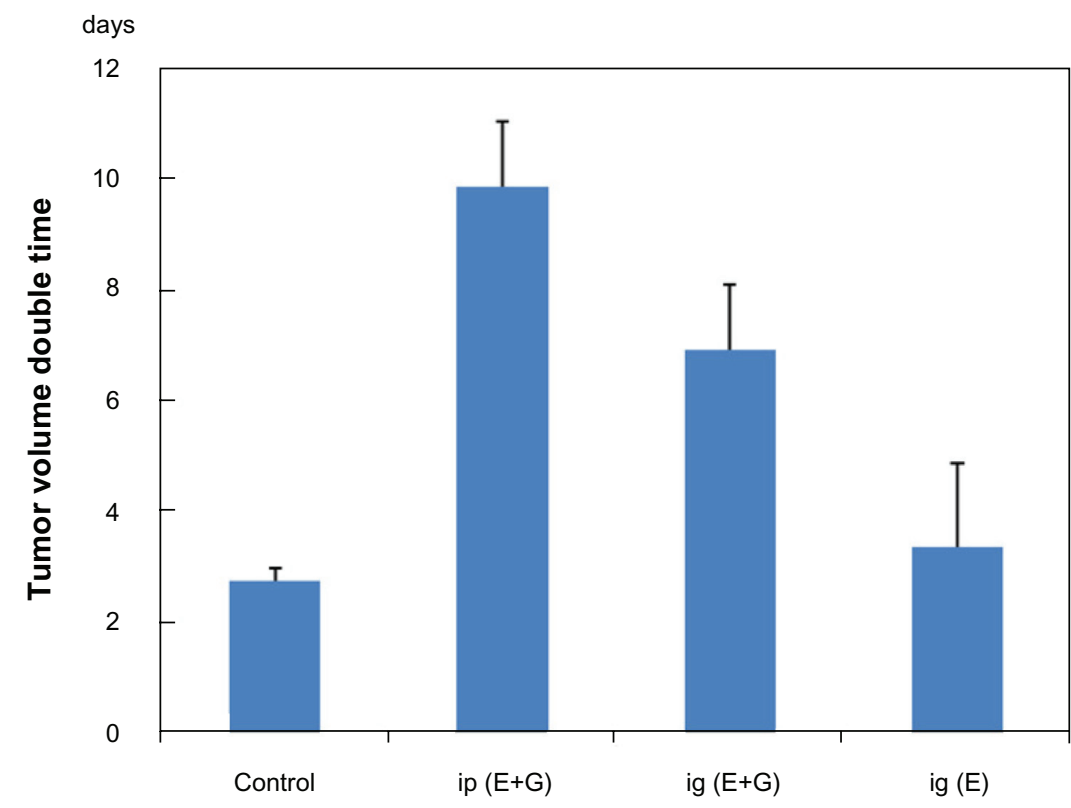

Figure 6 (Continued) 


\section{C}

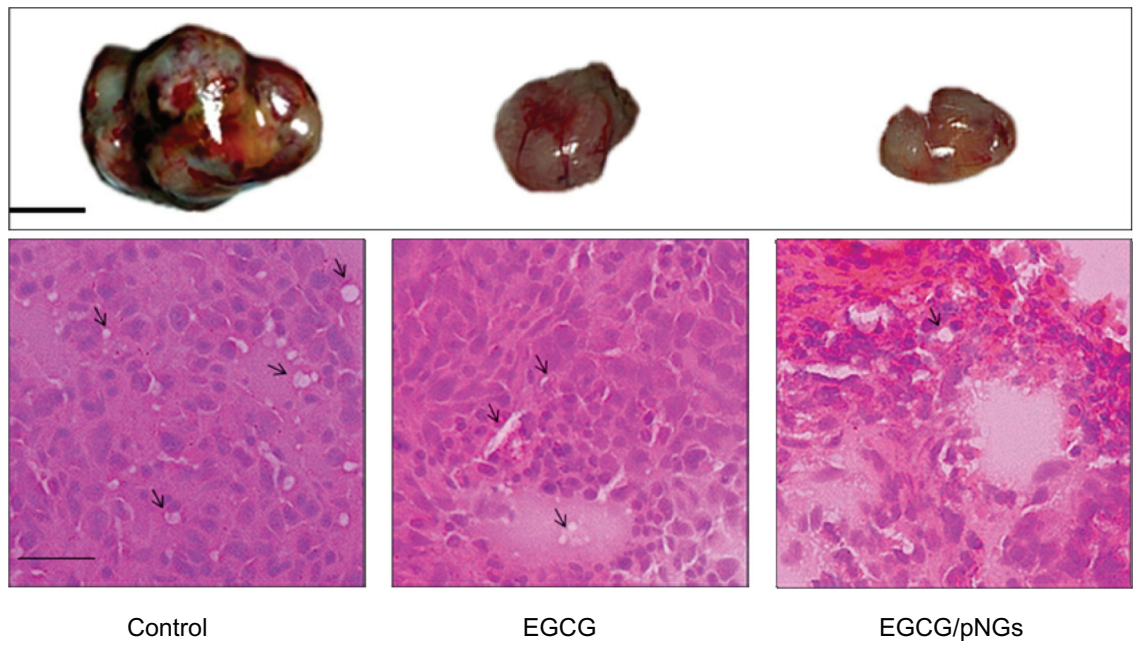

Figure 6 Growth inhibition effects of EGCG and in combination with pNG in a murine MBT-2 subcutaneous tumor model: (A) tumor-volume ratio in the mice was detected individually following treatment by feeding $2 \mathrm{mg} / \mathrm{mouse}$ EGCG or $2 \mathrm{mg} / \mathrm{mouse}$ EGCG-pNG (23:2.5) via an oral route (ig) and intaperitonially (ip); (B) tumor-volume double time indicating tumor volumes in mice receiving the different drugs (control, EGCG, and EGCG-pNG) at day 29; (C) photographs and histology (H\&E staining, 40×) of excised tumors from mice. Pictures of tumors show engorgement of tumor vessels in the control group that are larger than the EGCG and EGCG-pNG groups. The H\&E-staining histology of the tumor mass shows more vessels in the cross-sectional view in the control compared to the other groups.

Notes: Black line, I cm; black arrows, tumor vessels; scale bar, $25 \mu \mathrm{m}$.

Abbreviations: EGCG, epigallocatechin-3-gallate; H\&E, hematoxylin and eosin; pNG, physical nanogold.

with EGCG or control only. Less fluid accumulation in the peritoneal cavity was observed in EGCG-pNG-treated samples compared with the control and EGCG samples. As the size of the tumors was suppressed over 29 days after repeated oral administration in contrast to free EGCG, it was confirmed that EGCG-pNG had significant antitumor activities (Figure 6C).

The anticancer activity of EGCG-pNG was investigated in $\mathrm{C} 3 \mathrm{H} / \mathrm{HeN}$ mice subcutaneously implanted with MBT-2 murine bladder tumor cells. EGCG-pNG was confirmed to inhibit tumor cell growth by means of cell apoptosis. ${ }^{9}$ The mechanism by which EGCG-pNG mediated tumor apoptosis was demonstrated to involve activation of the caspase cascade, via the Bcl-family proteins, of the mitochondrial pathway. Additionally, the mechanism by which tumors were suppressed following injection of EGCG-pNG directly into the tumor site was determined to be through downregulation of vascular endothelial growth factor, whereas the effect following oral administration of EGCG was through reversing immune suppression upon cancer progression. The prepared EGCG-pNG was confirmed to be more effective than free EGCG in inhibiting bladder tumors in the mouse model. Mukherjee et $\mathrm{al}^{24}$ reported that AuNPs can interact specifically to bind vascular permeability factor/vascular endothelial growth factor and basic fibroblast growth factor, two endothelial cell mitogens and mediators of angiogenesis, resulting in inhibition of endothelial/ fibroblast cell proliferation in vitro, induced permeability, and angiogenesis in vivo.

Because metal poisoning is associated with renal and hepatic toxicities, we determined the effect of nanogold and EGCG-pNG administration on liver and renal functions. Normal mice were given the same dose of nanogold and EGCG-AuNPs as in the mouse tumor model for 7 consecutive days. On day 8 , the mice were killed and serum was collected. There were no significant differences between serum levels of creatinine, blood urea nitrogen, bilirubin alkaline phosphatase, alanine aminotransferase, or aspartate aminotransferase between the EGCG-pNG or nanogoldtreated and untreated control animals (data not shown).

Physical nanogold has been extensively used for a variety of biomedical applications and has been employed not only as a targeted contrast agent but as scaffolding for cancer drug delivery, a transfection agent for gene therapy, and an intrinsic antineoplastic agent. ${ }^{25}$ Portney et al employed a cowpea mosaic virus to form NG-CPMV hybrids that were used to generate fluorescence for image enhancement in MRI. ${ }^{26}$ Zheng et al demonstrated the utility of ferritin bioconjugated nanogold for biomedical detection via surface plasmon resonance. ${ }^{27}$ Mandal and colleagues also reported the use of AuNPs chemically cross-linked to carrier peptides for directed subcellular localization in osteosarcoma. ${ }^{28}$ In this current study, we presented nanogold hybrid self-assembly via ultrasound homogenization and showed that $\mathrm{pNG}$ could 
be successfully employed to deliver EGCG for prolonged antitumor and antioxidant activity.

\section{Conclusion}

We reported that size-controlled EGCG-pNG particles exhibited stability over a wide range of lower $\mathrm{pH}$ levels, and thus could adapt to gastric digestion. Furthermore, we have demonstrated the applicability of these nanoparticles as carriers for the delivery of anticancer drugs to enhance antioxidant activity and antitumorigenesis. EGCG-pNG particles showed great potential for the creation of drugdelivery systems, as their stability and low toxicity offers many possibilities for further developing the formulation of EGCG for medical applications.

\section{Acknowledgments}

We would like to thank the National Science Council (NSC 98-2320-B-016-003-MY3) and the Tri-Service General Hospital (TSGH-C98-100) for partial financial support.

\section{Disclosure}

The authors report no conflict of interest in this work.

\section{References}

1. Ghosh P, Han G, De M, Kim CK, Rotello VM. Gold nanoparticles in delivery applications. Adv Drug Deliv Rev. 2008;60(11):1307-1315.

2. Duncan B, Kim C, Rotello VM. Gold nanoparticle platforms as drug and biomacromolecule delivery systems. J Control Release. 2010;148(1): 122-127.

3. Cai YJ, Ma LP, Hou LF, Zhou B, Yang L, Liu ZL. Antioxidant effects of green tea polyphenols on free radical initiated peroxidation of rat liver microsomes. Chem Phys Lipids. 2002;120(1-2):109-117.

4. Butt MS, Sultan MT. Green tea: nature's defense against malignancies. Crit Rev Food Sci Nutr. 2009;49(5):463-473.

5. Kuroda Y, Hara Y. Antimutagenic and anticarcinogenic activity of tea polyphenols. Mutat Res. 1999;436(1):69-97.

6. Dai F, Chen WF, Zhou B. Antioxidant synergism of green tea polyphenols with a-tocopherol and L-ascorbic acid in SDS micelles. Biochimie. 2008; 90(10): 1499-1505.

7. Ryan P, Hynes MJ. The kinetics and mechanisms of the complex formation and antioxidant behaviour of the polyphenols EGCg and ECG with iron(III). J Inorg Biochem. 2007;101(4):585-593.

8. Navarro RE, Santacruz H, Inoue M. Complexation of epigallocatechin gallate (a green tea extract, egcg) with $\mathrm{Mn}^{2+}$ : nuclear spin relaxation by the paramagnetic ion. J Inorg Biochem. 2005;99(2):584-588.

9. Hsieh DS, Wang H, Tan SW, et al. The treatment of bladder cancer in a mouse model by epigallocatechin-3-gallate gold nanoparticles. Biomaterials. 2011;32(30):7633-7640.

International Journal of Nanomedicine

\section{Publish your work in this journal}

The International Journal of Nanomedicine is an international, peerreviewed journal focusing on the application of nanotechnology in diagnostics, therapeutics, and drug delivery systems throughout the biomedical field. This journal is indexed on PubMed Central, MedLine, CAS, SciSearch ${ }^{\circledR}$, Current Contents $₫ /$ Clinical Medicine,
10. Song WS, Jeon DG, Kong CB, et al. Tumor volume of increase during preoperative chemotherapy as a novel predictor of local recurrence in extremity osteosarcoma. Ann Surg Oncol. 2011;18:1710-1716.

11. Fujimaki T, Matsuno A, Sasaki T, et al. Proliferative activity of central neurocytoma: measurement of tumor volume doubling time, MIB-1staining index and bromodeoxyuridine labeling index. $J$ Neurooncol. 1997;32:103-109.

12. Bhumkar D, Joshi H, Sastry M, Pokharkar V. Chitosan reduced gold nanoparticles as novel carriers for transmucosal delivery of insulin. Pharm Res. 2007;24(8):1415-1426.

13. Wisse E, Braet F, Luo D, et al. Structure and function of sinusoidal lining cells in the liver. Toxicol Pathol. 1996;24(1):100-111.

14. Yuan F, Dellian M, Fukumura D, et al. Vascular permeability in a human tumor xenograft: molecular size dependence and cutoff size. Cancer Res. 1995;55:3752-3756.

15. Sonavane G, Tomoda K, Makinoa K. Biodistribution of colloidal gold nanoparticles after intravenous administration: effect of particle size. Colloids Surf B Biointerfaces. 2008;66(2):274-280.

16. Rouhana LL, Jaber JA, Schlenoff JB. Aggregation-resistant watersoluble gold nanoparticles. Langmuir. 2007;23(26):12799-12801.

17. Chen LS, Lee MJ, Li HE, Yang CS. Absorption, distribution, and elimination of tea polyphenols in rats. Drug Metab Dispos. 1997;25(9): $1045-1050$.

18. Chen EP, Mahar Doan KM, Portelli S, Coatney R, Vaden V, Shi W. Gastric $\mathrm{pH}$ and gastric residence time in fasted and fed conscious cynomolgus monkeys using the Bravo $\mathrm{pH}$ system. Pharm Res. 2008; 25(1):123-134.

19. Dressman JB, Berardi RR, Dermentzoglou LC, et al. Upper gastrointestinal (GI) $\mathrm{pH}$ in young, healthy men and women. Pharm Res. 1990;7(7):756-761.

20. Venkatpurwar V, Shiras A, Pokharkar V. Porphyran capped gold nanoparticles as a novel carrier for delivery of anticancer drug: In vitro cytotoxicity study. Int J Pharm. 2011;409(1-2):314-320.

21. Guo D, Wu C, Song W, Jiang H, Wang X, Chen B. Effect of colloidal gold nanoparticles on cell interface and their enhanced intracellular uptake of arsenic trioxide in leukemia cancer cells. J Nanosci Nanotechnol. 2009;9(8):4611-4617.

22. Chen YH, Tsai CY, Huang PY, et al. Methotrexate conjugated to gold nanoparticles inhibits tumor growth in a syngeneic lung tumor model. Mol Pharm. 2007;4(5):713-722.

23. Dhar S, Reddy EM, Shiras A, Pokharkar V, Prasad BL. Natural gum reduced/stabilized gold nanoparticles for drug delivery formulations. Chemistry. 2008;14(33):10244-10250.

24. Mukherjee $P$, Bhattacharya $R$, Wang $P$, et al. Antiangiogenic properties of gold nanoparticles. Clin Cancer Res. 2005;11(9):3530-3534.

25. Dreaden EC, Mackey MA, Huang X, Kang B, El-Sayed MA. Beating cancer in multiple ways using nanogold. Chem Soc Rev. 2011;40(7): 3391-3404.

26. Portney NG, Destito G, Manchester M, Ozkan M. Hybrid assembly of CPMV viruses and surface characteristics of different mutants. Curr Top Microbiol Immunol. 2009;327:59-69.

27. Zheng B, Yamashita I, Uenuma M, Iwahori K, Kobayashi M, Uraoka Y. Site-directed delivery of ferritin-encapsulated gold nanoparticles. Nanotechnology. 2010;21(4):045305.

28. Mandal D, Maran A, Yaszemski MJ, Bolander ME, Sarkar G. Cellular uptake of gold nanoparticles directly cross-linked with carrier peptides by osteosarcoma cells. J Mater Sci Mater Med. 2009;20(1):347-350.

\section{Dovepress}

Journal Citation Reports/Science Edition, EMBase, Scopus and the Elsevier Bibliographic databases. The manuscript management system is completely online and includes a very quick and fair peer-review system, which is all easy to use. Visit http://www.dovepress.com/ testimonials.php to read real quotes from published authors. 